Meta

Journal des traducteurs

Translators' Journal

\title{
La linguistique et l'informatique, des alliées
}

\section{Conrad Ouellon}

Volume 34, numéro 3, septembre 1989

1. Actes du Colloque Les terminologies spécialisées : Approches quantitative et logico-sémantique et 2 . Actes du Colloque Terminologie et Industries de la langue

URI : https://id.erudit.org/iderudit/003773ar

DOI : https://doi.org/10.7202/003773ar

Aller au sommaire du numéro

Éditeur(s)

Les Presses de l'Université de Montréal

ISSN

0026-0452 (imprimé)

1492-1421 (numérique)

Découvrir la revue

Citer cet article

Ouellon, C. (1989). La linguistique et l'informatique, des alliées. Meta, 34(3),

552-557. https://doi.org/10.7202/003773ar d'utilisation que vous pouvez consulter en ligne.

https://apropos.erudit.org/fr/usagers/politique-dutilisation/ 


\title{
LA LINGUISTIQUE ET L'INFORMATIQUE, DES ALLIÉES
}

\author{
Conrad Ouellon \\ $C I R B$ \\ Université Laval, Québec, Canada
}

L'adaptation de la langue française au marché des langues, les rapports que la langue doit entretenir avec la nouvelle société technologique, la place du français dans l'univers informatique, voilà autant de questions qui préoccupent actuellement les gouvernements des pays francophones. La reconnaissance du français comme langue de culture scientifique devra bien sûr s'appuyer sur une volonté politique clairement affirmée des chefs d'état; il faudra aussi, et surtout, que les spécialistes de la langue, les linguistes en particulier, réfléchissent au rôle qu'ils pourraient jouer dans une société où le concept des industries de la langue s'impose de plus en plus.

Quels sont les obstacles que devront surmonter les linguistes et les langagiers, quelles attitudes nouvelles devront-ils adopter pour participer pleinement à l'effort de francisation du monde technologique? Il n'existe pas de réponse universelle et catégorique à ces interrogations. Je pense toutefois que les commentaires et réflexions qui suivent, même s'ils sont inspirés d'une expérience particulière qu'a vécu le Département de langues et linguistique de l'Université Laval au cours des trois dernières années, valent pour d'autres groupes de linguistes, en milieu universitaire tout particulièrement. $\mathrm{Ce}$ département, en effet, est à l'image de plusieurs autres départements ce qui rend mes propos généralisables.

\section{L'EXPÉRIENCE IBM / LAVAL}

\section{A) LA SITUATION ORIGINELLE DE L'ORGANISATION}

Avant de décrire l'expérience que notre département a vécue, il faut faire une brève description de la situation de l'unité en 1985, il y a donc trois ans à peine...

Le département de langues et linguistique de l'Université Laval est, à ma connaissance, l'un des plus imposants départements canadiens de linguistique; il peut compter sur 59 postes réguliers de professeurs et dispense l'enseignement à un équivalent d'environ 850 étudiants à temps plein au premier cycle. Plus de 150 étudiants ont un dossier actif aux études de $2 \mathrm{e}$ et $3 \mathrm{e}$ cycles. Nous intervenons dans de nombreux programmes, dont les plus importants sont ceux de linguistique française, de traduction, d'enseignement du français langue seconde. Les principaux projets de recherche du département en 1985 étaient le Trésor de la langue française au Québec, les études sociolinguistiques sur les variétés de français parlées à Québec, les relevés des langues du monde du CIRB (Centre international de recherches sur l'aménagement linguistique), l'édition des œuvres de Gustave Guillaume.

Du point de vue théorique, le département était connu pour son attachement à la psychomécanique du langage de Gustave Guillaume. Les autres courants théoriques existaient sans que leur importance soit très bien reconnue. Bref, c'était un département ouvert à de multiples courants, plutôt théorisant, sans rapport bien défini avec la société industrielle, avec l'industrie tout court. 
Nos contacts avec le monde technologiques, avec la technique, étaient minces; du point de vue matériel, le département disposait tout au plus d'un laboratoire de phonétique équipé pour la recherche en phonétique articulatoire surtout. Pour tout équipement informatique, le département ne possédait que de trois micro-ordinateurs, tous affectés à des fins de recherche.

Nous étions par ailleurs fort peu préoccupés par le statut du français langue de communication et de culture scientifiques à l'époque.

\section{B) L'ÉVÉNEMENT IBM-LAVAL}

En 1985, le Département de langues et linguistique était invité à participer à un projet de collaboration avec la Société IBM / Canada de même qu'avec le Département d'informatique et la Faculté des sciences de l'administration.

Le Département de langues et linguistique n'a disposé que d'une fin de semaine pour présenter un projet sommaire de recherche; il n'est guère important pour les fins de cet exposé de donner le détail de ce projet, je me contenterai de dire qu'il nous apparaît maintenant un peu naiif.

Derrière ce projet toutefois, il y avait une volonté politique bien claire, celle de provoquer des rapprochements entre les théoriciens et les praticiens, entre si l'on veut, les linguistes d'une part et les didacticiens, traducteurs et terminologues d'autre part. C'est le genre de divisions qui existent dans d'autres universités. Au delà du projet déposé, nous visions aussi, et c'était un préalable à la réussite du projet de recherche, à rapprocher linguistique et informatique, c'est-à-dire à faire connaître aux linguistes les avantages... et limites... de l'ordinateur, à faire aussi voir aux informaticiens et autres utilisateurs traditionnels de l'ordinateur (de diverses organisations) les avantages qu'ils retireraient à recourir à des spécialistes de la langue, surtout en contexte d'informatisation en langue française.

En décembre 1985, une entente de collaboration de trois ans entre la Société IBM et l'université Laval était enfin signée. Du même coup, le département de langues et linguistique voyait son parc informatique passer de trois à quarante micro-ordinateurs et disposait en plus d'un budget de recherches de $.64 \mathrm{M}$ à utilisation très souple. Les appareils étaient répartis à parts égales pour des fins d'enseignement (1 $1^{\text {er }}$ cycle en priorité) et de recherche. L'ensemble était lié en réseau, ce qui permettrait plus tard les télécommunications avec des centres universitaires du monde entier (NetNorth, BitNet et EARN). Quinze professeurs étaient activement impliqués dans le projet; leur adhésion avait été obtenue après qu'ils eussent, évidemment, reçu la garantie qu'ils n'avaient aucune obligation envers la Société IBM autre que celle de rendre public (par des publications, communications, conférences, etc.) tout ce qui serait réalisé dans les cadres de l'entente.

En vue d'assurer le succès de l'opération informatisation, nous y avons associé les étudiants de très près. Ils forcèrent d'une certaine façon le corps professoral et le département à s'ajuster à la nouvelle réalité par la création d'une forte demande de services de leur part.

Évidemment, l'implantation de l'informatique ne s'est pas faite facilement en bonne partie parce que rares étaient les professeurs capables d'encadrer les étudiants, en mesure d'orienter les efforts. On peut même penser que certains n'ont accepté de collaborer qu'après s'être assurés que nous n'allions pas vers une catastrophe.

Nous pouvons maintenant penser que notre projet a atteint ses objectifs. Le département, réputé «théorisant» et «traditionaliste», s'est suffisamment transformé pour qu'on le reconnaisse comme un interlocuteur valable dans le secteur des industries de la langue. 


\section{C) LES RÉSULTATS DE L'EXPÉRIENCE}

$\mathrm{Si}$ on laisse de côté les aspects purement matériels ou techniques, ce projet a-t-il modifié les attitudes du département devant les besoins de la société, devant les mutations technologiques en cours? Nous pouvons sans hésitation répondre affirmativement, sans que se soient cependant effacées les habituelles réactions au changement.

1) En premier lieu, de nouveaux champs de recherche se sont ouverts. Les questions touchant l'informatique et la linguistique sont devenues à la mode. On ne refuse plus de s'intéresser à des projets de recherche qui visent ultimement la production d'outils comme des didacticiels, des logiciels d'aide à la rédaction, comme des dictionnaires, des lexiques, des terminologies bilingues ou non, comme des logiciels pour la reconnaissance automatique assistée de termes simples ou complexes. Les phonéticiens travaillent activement au développement de connaissances qui permettent la production d'une parole de synthèse adaptée au milieu d'implantation du système. Bref, plusieurs professeurs et chercheurs s'intéressent maintenant au transfert de leurs connaissances en vue d'applications concrètes, en langue française surtout.

2) Les étudiants de tous les cycles démontrent un vif intérêt pour ces nouveaux domaines d'études et de recherche. Le taux de fréquentation des laboratoires est très élevé. Les étudiants y ont trouvé un champ d'étude qui semble pouvoir déboucher sur un emploi. Plusieurs avouent une plus grande motivation et découvrent des possibilités nouvelles d'un travail intéressant. Ces étudiants discutent maintenant de technique, de logiciel, de linguistique aussi... Terminologues, traducteurs, linguistes discutent entre eux et collaborent dans les mêmes projets.

3) Le projet a également créé une nouvelle dynamique dans la structure administrative.

a) Les programmes comme le baccalauréat en traduction ont été les premiers évidemment à profiter des nouvelles facilités informatiques: traitement de textes, interrogation en direct des banques de terminologie (TERMIUN, BTQ, IBMot). L'initiation à l'informatique est devenue obligatoire dans ces programmes. Les étudiants sont très conscients des avantages qu'ils retireront, qu'ils retirent déjà de ces connaissances dans leur quête d'un emploi.

b) Les programmes de linguistique française et générale sont en voie de révision et tiendront compte des nouvelles possiblités de recherche et des techniques dont nous disposons maintenant.

c) Le CIRB (Centre international de recherche en aménagement linguistique) est en voie de redéfinir sa vocation de recherche. L'un des axes principaux de réflexion qu'il retient, ce sont les études qui permettront à la société francophone, québécoise tout particulièrement, de faire face dans sa langue à l'informatisation massive de la société et du monde du travail. Préoccupation d'autant plus importante qu'une récente enquête du Conseil de la langue française du Québec laisse entendre que le retard du Québec par rapport aux autres provinces du Canada dans l'informatisation serait dû en partie à la méconnaissance de la langue anglaise de la plus grande partie de la population. Le CIRB pense aussi, comme d'autres, que le français doit devenir une langue traitable par l'ordinateur, que les outils de production de textes, les aides à la rédaction, les terminologies, les lexiques, etc doivent devenir très rapidement disponibles si on tient véritablement à ce que le français demeure une langue véhiculaire plutôt qu'une langue réservée aux loisirs, à l'expression de la culture.

D) LES OBSTACLES AU DÉVELOPPEMENT

Il est cependant bien clair que cette transformation du département ou si l'on veut, ce développement d'un important champ d'activités comme les «industries de la langue» se bute toujours à quelques obstacles : 
1) Les étudiants issus pour la plupart des sciences humaines ont des préjugés devant l'ordinateur; ils s'imaginent qu'il faut être fort en sciences pour utiliser l'informatique. Les professeurs, quant à eux, n'admettent pas tous la nécessité de l'informatique, plusieurs n'y voyant qu'une dactylo améliorée. Ils ne s'opposent toutefois plus au changement en cours.

2) La lourdeur administrative de l'institution universitaire rend difficile la modernisation de nos programmes, ainsi que je l'ai dit plus haut. Il faudrait qu'on puisse rapidement mettre sur pied un cheminement de programme de linguistique informatique dont les cours seraient ouverts à d'autres programmes.

3) Le faible taux de renouvellement du corps professoral et l'impossibilité virtuelle de recyclage de quelques professeurs en industries de la langue constituent un autre obstacle majeur. Il faut en arriver à créer un besoin, à se placer dans l'impossiblité d'y répondre pour réussir à convaincre de la nécessité d'embaucher un autre spécialiste en informatique linguistique, par exemple.

4) Nos administrateurs craignent, à tort souvent, de ne pouvoir entretenir et renouveler le parc informatique. On n'est en effet pas toujours convaincu que les étudiants de lettres ont besoin d'autre chose que de crayons et de papier... Corollaire de cette crainte, la difficulté d'obtenir le soutien technique approprié.

\section{RÉFLEXIONS SUSCITÉES PAR L'EXPÉRIENCE}

Ce compte-rendu de l'expérience que nous vivons permet de dégager quelques points intéressants quant au comportement que devraient avoir les spécialistes de la langue, les linguistés, devant la société technologique, devant les difficultés que rencontre le français à s'imposer dans le discours technique, dans les nouvelles technologies.

Les multiples contacts que nous avons eus avec des sociétés privées, d'abord avec la multinationale IBM, les relations au début orageuses que nous avons établies avec des collègues de sciences de l'administration, d'informatique, les rencontres avec des consommateurs de produits informatiques ont modifié nos attitudes traditionnelles de linguiste.

1) En tout premier lieu, nous avons rapidement constaté que les non linguistes ne nous comprenaient pas. Notre jargon, il faut bien le reconnaître, limite l'auditoire à nos seuls collègues. C'est à nous qu'il revient de vendre notre produit ou, dit autrement pour ceux qui trouveraient un peu trop mercantile l'expression, de démontrer à quoi peut servir la linguistique, la terminologie, la phonétique, notre connaissance de la langue. Dans le contexte du marché des langues, nous sommes des vendeurs. Nous avons à faire la preuve de la valeur de notre produit.

2) En second lieu, nous avons constaté, au contact de collègues d'autres disciplines, combien nous étions éloignés des préoccupations d'une couche importante de la société qui nous entoure. Et j'ai l'impression que ces collègues, de sciences de l'administration, d'informatique, ont, entre autres mérites, celui d'être souvent plus près que nous de cette société...

3) En troisième lieu, il est plutôt stimulant de s'apercevoir que notre science peut être utile, que des spécialistes d'autres disciplines croient qu'elle peut l'être, y croient suffisamment pour nous inviter à nous intégrer à leurs équipes...

4) Enfin, nous avons réalisé qu'il était possible de concilier technique et théorie, recherche fondamentale et recherche appliquée... Notre équipe a déposé au cours des derniers mois d'importantes demandes de subventions pour des projets d'industries de la langue. 


\section{CONCLUSIONS}

Quelles sont les conditions qu'il faut réunir pour assurer la communication entre le marché des langues et la linguistique? Je vais en énumérer quelques-unes.

- Il faut d'abord faire preuve d'ouverture d'esprit. Autant que faire se peut, les programmes de linguistique devraient offrir des enseignements en littérature technique, en terminologie scientifique, en rédaction technique, en traitement des données, en linguistique informatique,... Il faudrait aussi qu'on cesse de perpétuer le mythe de l'anglais seule langue apte à traduire l'objet matériel, l'objet technique; le français a déjà rempli, et continue de le faire, les mêmes fonctions... Il faut aussi qu'on admette que la langue française, si noble, si pure soit-elle, cette langue faite pour exprimer de beaux sentiments, possède aussi les ressources pour rendre compte des techniques. On doit permettre que la langue française puisse devenir un objet technique, puisse même être manipulée pour devenir objet informatique, en attendant que l'informatique soit suffisamment développée pour saisir et traiter la langue telle quelle. Et les résistances à cette manipulation sont toujours présentes.

- L'Université, les universitaires surtout, doivent se mettre à l'écoute de la société. L'Université doit être à l'écoute des besoins de la société dont elle dépend. Il arrive toutefois fréquemment que ces besoins soient interprétés et filtrés par la machine universitaire; d'où une plus ou moins grande absence de correspondance entre le besoin social et la réponse de l'institution universitaire. Je reconnais que les filtres ont comme vertu d'éliminer ce qui relève de la mode, mais il arrive qu'en d'autres occasions ils laissent l'Université en marge de l'évolution. Dans le cas précis de l'adaptation à l'évolution technologique, les universités devront proposer des enseignements plus adaptés, valoriser la production scientifique en langue française. Sinon, on continuera de discourir sur la place que doit occuper la langue française dans les nouvelles technologies.

- On ne peut rien produire à partir de rien, même en sciences humaines. Il faudra donc que nos institutions investissent davantage dans le recrutement, le recyclage des professeurs; plus personne ne se fait d'illusions sur la volonté de changement des révolutionnaires de mai 1968 qui occupent aujourd'hui les postes de commande dans nos institutions. Les départements de linguistique devraient aussi pouvoir compter sur des ressources techniques comme l'informatique, si on veut que les étudiants profitent des nouvelles technologies, et fassent aussi bénéficier celles-ci de leur connaissance de la langue. Ce ne sont pas les gens de notre génération qui feront le virage informatique à l'Université, mais bien les étudiants que nous formons. Ils doivent recevoir une formation adaptée aux exigences de l'employeur, à l'appareillage disponible en milieu de travail.

- Il faut que les linguistes en arrivent à se faire comprendre des spécialistes des autres disciplines. Il faut aussi qu'on accepte de travailler avec ces spécialistes, qu'on profite de leur expérience, qu'on tienne compte de leurs sensibilités.

- Il faut qu'il y ait partage des connaissances entre linguistes des diverses universités des pays francophones, collaboration malgré les mesquineries universitaires, dans un climat de saine concurrence. Il ne sert à rien de rêver à l'impossible, mais notre influence n'en serait que plus forte si la coopération entre linguistes, terminologues, autres experts de la langue était plus évidente. En matière de langue toutefois, sujet émotif s'il en est, où chacun croit son opinion la meilleure, les visées communes sont rares !

- Enfin, les spécialistes de la langue française, s'ils veulent avoir leur mot à dire đans l'expression des nouvelles technologies, s'ils veulent être entendus en dehors de leur pays, de leur université, vont devoir tenir compte de la variation linguistique. Dans mon pays, en effet, la meilleure variété de langue, c'est celle qui y a cours ! J'imagine que chacun pense la même chose de sa variété de français...Ce n'est pas pour rien que IBMCanada et IBM-France ont des services de traduction et de terminologie distincts. La 
publicité ne se conçoit ni ne s'écrit de la même façon dans les deux pays. Les manuels d'informatique produits en France charrient des référents culturels qui ne sont pas nécessairement familiers à tous les Québécois. La variation linguistique existe si on admet la variation culturelle...

Pour terminer, je reprends l'opinion d'autres collègues intéressés au sort et à l'avenir de la langue française. L'adaptation de la langue française à la société technologique est peut-être devenue une condition de sa survie comme langue de travail, comme langue véhiculaire tout court. De toute façon, en tant que linguiste, je préfère intervenir activement dans le dossier des «industries de la langue» plutôt que d'assister comme observateur neutre à l'effacement de la langue française comme outil de communication et de travail dans cet important secteur de l'activité humaine marqué par les nouvelles technologies. 